\title{
Molecular Assessment of Glutathion S-Transferases (GSTT1 \& GSTM1) Genotypes in HCV Infection
}

\author{
Farmanullah* ${ }^{*}$, Rehana Yasmin ${ }^{1}$, Aaqib Shaheen ${ }^{1}$, Nawab Ali ${ }^{1}$, Muhammad Noaman Saeed ${ }^{1}$, \\ Muhammad Jamil ${ }^{1}$, Muhammad Saeed ${ }^{2}$ \\ ${ }^{1}$ Department of Biotechnology \& Genetic Engineering, Kohat University of Science \& Technology, Kohat, (Pakistan) \\ ${ }^{2}$ Department of Biosciences, COMSATS Institute of Information Technology, Islamabad, (Pakistan) \\ *Corresponding Author: tofarman@gmail.com
}

\begin{abstract}
Glutathione S-Transferases (GSTs) are super family phase II detoxification enzymes, which regulate oxidative stress. GST Mu-1 (GSTM1) and GST Theta-1 (GSTT1) are expressed frequently in liver cell and have been observed to have prominent role in neutralizing reactive oxygen species producing as results of normal metabolism, stress or infection. Oxidative stress plays an important role in the pathogenesis of $\mathrm{HCV}$-induced damage because GSTs directly involved in the detoxification of reactive oxygen species. Total 76 blood samples were collected. Various risk factors regarding HCV \& GSTs were evaluated. Human genomic DNA was extracted through phenol/chloroform method and GSTs genotype was analyzed observed. Among 56 patient's samples, GSTT1 (-), GSTM1 (-) and null genotype were $21.05 \%, 28.95 \%, 23.69 \%$ respectively while $26.3 \%$ of individuals showed normal GST pattern. Similarly among the male $(63.16 \%)$ the ratios of GSTT1(-), GSTM1(-) and null genotype were $11.84 \%, 17.11 \%$ and $18.42 \%$ respectively, while among total female (36.84\%) the ratios for GSTT1(-), GSTM1(-) and Null genotypes were $9.21 \%, 11.84 \%$ and $5.26 \%$ respectively. The individuals younger than 31 years showed GSTT1(-) $2.63 \%$, GSTM1(-) $6.58 \%$ and null genotype $3.95 \%$. While individuals elder than 31 years GSTT1(-) were $18.42 \%$ GSTM1(-), $22.37 \%$ and null genotypes were $19.74 \%$. It has been concluded that increase in age, increases the chances of both GSTT1 \& GSTM1 deletion(-), irrespective of null genotypes, however chronic HCV infection produce frequent GST's null genotypes. Among various risk factors tobacco addiction and unhygienic life standard seems to be the risk factors.
\end{abstract}

Keywords; GST, HCV, Null genotype, Oxidative stress.

\section{Introduction}

Glutathione S-Transferases, are major phase II detoxification enzymes which are involved in the metabolism of xenobiotics and play an important role in cellular protection against oxidative stress. The GSTs are a family of enzymes that catalyze the formation of thio-ether conjugates between the endogenous tripeptide glutathione and xenobiotic compounds [1]. The major biological function of glutathione transferases appears to be defense against reactive and toxic electrophiles such as reactive oxygen species (superoxide radical and hydrogen peroxide) that arise through normal metabolic processes. Many of these are formed by cellular oxidative reactions catalyzed by cytochrome $\mathrm{P}_{450}$ and other oxidases [2]. Two distinct superfamilies of GSTs have been described. One soluble GST superfamily is subdivided into eight separate classes designated Alpha, Kappa, Mu, Pi, Sigma, Theta, Zeta and Omega. Soluble GSTs have been described 
mainly in cytoplasm but they are also presented in nucleus, mitochondria and peroxisomes while other super family of GSTs designated as membraneassociated proteins in eicosanoid and glutathione metabolism (MAPEG) probably with trimeric structure, is involved in arachidonic acid metabolism [1].Soluble GSTs and MAPEG are widely distributed throughout the body tissues more specifically in liver [3]. Several types of allelic variations have been identified in the class Alpha, Mu, Pi, Theta GST gene families. Individuals lacking GST-M1, GST-T1 and GST-P1 genes have a higher incidence of liver, bladder, breast, colorectal, head/neck and lung cancer. Loss of these genes has also been found to increase susceptibility to liver diseases, asthma and allergies, atherosclerosis and rheumatoid arthritis [4]. About 150 million individuals are chronically infected with hepatitis $\mathrm{C}$ virus (HCV) throughout the world [5]. While in Pakistan about 10 million peoples are infected from HCV infection [6]. Oxidative stress plays an important role in the pathogenesis of $\mathrm{HCV}$ induced damage [7] Glutathione (GSH) is the main element of the endogenous antioxidant system, which prevents the accumulation of free radicals through the transformation of GSH, catalyzed by glutathione peroxidase, into dimeric oxidized glutathione, which is converted back to GSH by glutathione s-transferse (GST) [8]. The most extensively studied GST isoforms in relation to the risk of environment-related human diseases are GSTM1 and GSTT1. GSTM1 and GSTT1 enzymes widely expressed in hepatocytes [9] and in Kupffer cells [10]. These have been identified in the cytosol and in mitochondria, where the rate of production of free radicals is highest. Available evidence indicates that GST isozymes, including the M1 and $\mathrm{T} 1$ isoforms, are involved in the detoxification of 4-hydroxy-alkenals, among them 9-hydroxynonenal [11]. The GST mu 1 (GSTM1) gene and the GST theta 1 (GSTT1) gene frequently have a partial deletion that causes the total absence of enzymatic function (null alleles). Both polymorphic traits are inherited independently. Individuals homozygous for null alleles of either gene are devoid of specific enzymatic activity in all their organs and tissues [12]. Moreover, GSTT1 has glutathione-peroxidase activity, and it might be directly involved in the detoxification of reactive species produced as a consequence of ongoing $\mathrm{HCV}$ infection of the liver. In chronic hepatitis $\mathrm{C}$, there is an accelerated turnover of GSH [13]. Glutathion S Transferase has been reported to play a major role in HCV infection [14]. Therefore it can be postulated that individuals devoid of GST enzyme activity would be more likely to develop severe $\mathrm{HCV}$ infection.

\section{Methodology}

\section{Samples Collection}

Total 76 blood samples were collected from the patients, who visited DHQ hospital Kohat. Among these recruited patients 56 samples were PCR positive while 20 samples were PCR negative, similarly among total $48(63.16 \%)$ were male and 28 (36.84\%) were female patients. At the time of sampling patients were interviewed and standard questionnaires were filled from their laboratory reports, clinical and histo-pathological reports through the concerned physicians. The questionnaires were included patient details i.e. age, socioeconomic status, risk factors, duration of infection, racial origin and location and living habitat. The socio-economic status criteria defined for this study is, those patients having a total monthly income less than 100 US\$ were considered as lower class, and more than 100 US\$ of middle class. After sampling blood was kept 
in EDTA vacutainer, transported to the department of biotechnology and genetic engineering KUST laboratory and stored at $-80{ }^{\circ} \mathrm{C}$ for further detailed analysis.

\section{DNA Extraction and Purification}

Human genomic DNA was extracted from all recruited samples for the amplification of GSTs specific gene by established laboratory DNA extraction protocol and purified the extracted genomic DNA by treating it with Phenol Chloroform Isoamylalcohol (PCI) solution repeatedly.

Molecular Analysis of Glutathione S-Transferases (GSTs)

For the amplification of GSTs, GST specific primers (Table. 1), GSTT1 and GSTM1 and $\beta$-Globulin (internal control) were used by performing multiplex PCR system [15]. A mixture of $25 \mu$ l was used for PCR reaction in a single PCR tube. PCR products were electrophoresed in $2 \%$ agarose gel and GSTT1 and GSTMI genotypes were determined by comparing the amplified product of genotypes with $\beta$ Globulin gene, used as internal standard and 100-bp DNA ladder (Fermantas USA), used as DNA size marker.

\section{Results and Discussion}

Among the total recruited patients, 62 (81.58\%) patients were at the age of greater than 31 years while $14(18.42 \%)$ patients were at the age of less than 31 years. Therefore from the groups of $\geq 31$ years old, $46(74.19 \%)$ patients were with PCR positive results that said to be a true patient of HCV while 16 (25.81 $\%)$ patients were with PCR negative results that said to be normal individuals. Similarly from the group of $\leq 31$ years old, there were $10(71.42 \%)$ patients having PCR positive results so placed as the true HCV patients while $4(28.57 \%)$ patients were with PCR negative therefore placed as normal individuals.
Similarly among the selected groups of under studying patients, $60(78.95 \%)$ were married while $16(21.05 \%)$ were unmarried. Among the married patients $46(76.66 \%)$ samples were with PCR positive patients while $14(23.33 \%)$ samples were normal individuals. However among the unmarried patients $10(62.5 \%)$ samples were with PCR positive patients while $6(37.5 \%)$ sample having PCR negative results, said to be normal individuals. Similarly among the various economical status 66 $(86.84 \%)$ individuals were, having Income $\leq 100$ US\$ (lower class), among these 49 (74.25\%) sample were PCR positive while 17 (25.75\%) samples were PCR negative, however 10 (13.16\%) individuals were, having income $\geq 100$ US\$ (middle class), among these 7 (70 \%) sample have PCR positive results while 3 (30\%) samples having PCR negative. Among the various potential risk factor proposed to associate risk of $\mathrm{HCV}$ infection with GSTs phase II metabolic enzymes involved in the neutralization of various carcinogens and to tolerate antiviral treatment for $\mathrm{HCV}$ included source of drinking water, Well (34.21\%), Tube well (44.74\%) and Tap water using individuals were $(21.05 \%)$. Similarly the individual who were drug adductors were 18 (23.68\%) persons of the total under studying group, however chain smoker were $58(76.32 \%)$ of the total individuals. Smoking is also previously reported as critical risk factor for the progress of tumor [16]. Acute and chronic infection classification, which has been done on the bases of investigation duration, serum ALT level, total viral load, liver ultrasound, histopathological report and duration and types of therapy for curing of $\mathrm{HCV}$ infection. Therefore on these bases, the total chronic $\mathrm{HCV}$ patients were 34 $(60.71 \%)$ and total acute HCV patients were 22 (39.29\%) (figure.4). There is no such patient having 
Table 1. Primers used for GSTT1, GSTM1 and $\beta$-globin (internal control).

\begin{tabular}{|c|c|c|}
\hline Genes & Polarity & Nucleotide Sequence (5' to 3') \\
\hline \multirow{2}{*}{ GSTT1 } & Sense & TTCCTTACTGGTCCTCACATCTC \\
& Antisense & TCACCGGATCATGGCCAGCA \\
\cline { 2 - 3 } & Sense & GAACTCCCTGAAAAGCTAAAGC \\
\hline \multirow{2}{*}{ GSTM1 } & Antisense & GTTGGGCTCAAATATACGGTGG \\
\cline { 2 - 3 } & Sense & GAAGAGCCAAGGACAGGTAC \\
\cline { 2 - 3 } & Antisense & CAACTTCATCCACGTTCACC \\
\hline
\end{tabular}

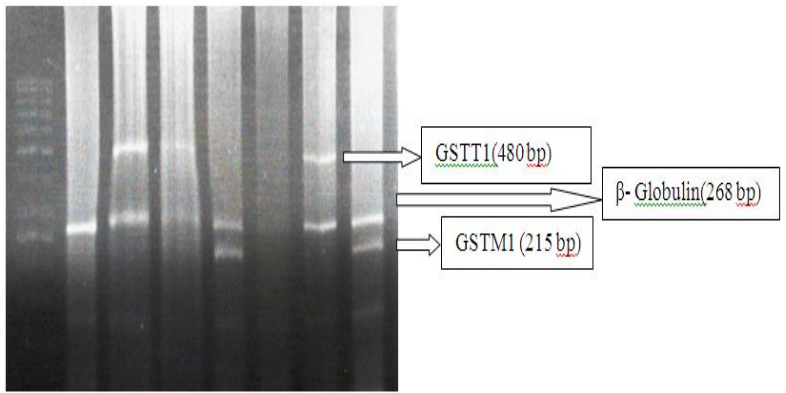

Figure 1. Gel electrophoreses of amplified GSTs genotypes.

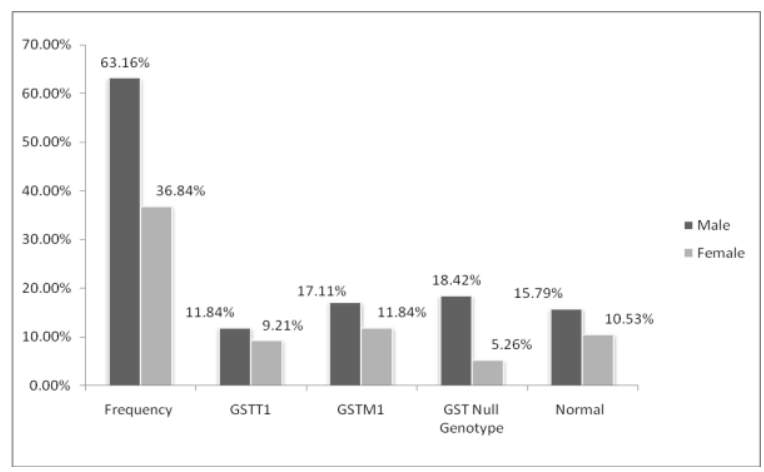

Figure 2. Gender wise percentage distribution of GSTs among male, female and normal individuals $(n=76, P$ value $\leq 0.05 \mathrm{~S})$.

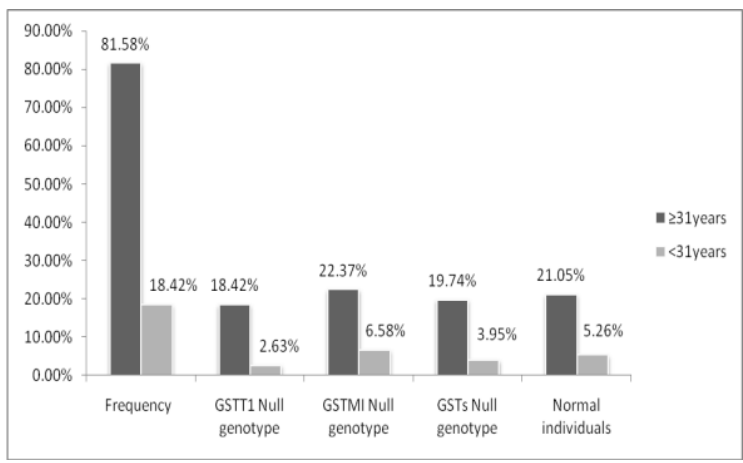

Figure 3. Age wise percentage distribution of GSTs among male, female and normal individuals ( $\mathrm{n}=76, \mathrm{~T}$ Test $\mathrm{P}$ value $\leq 0.05 \mathrm{~S}$ ).

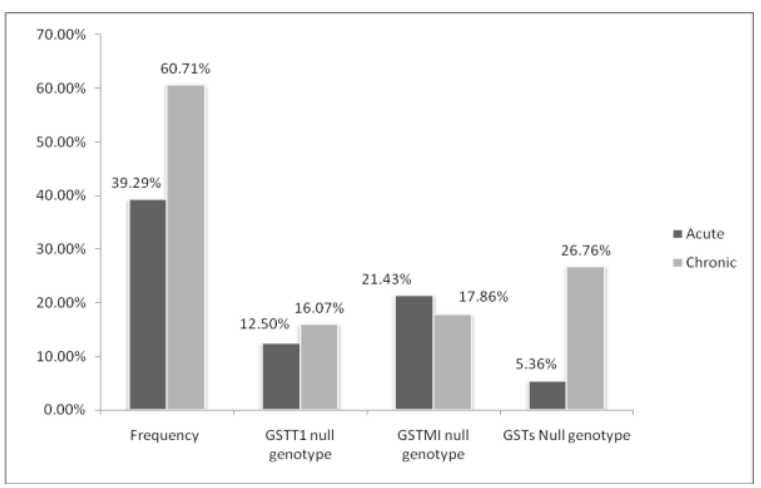

Figure 4. Infection wise percentage distribution of GSTs among the patients ( $n=56, T$ Test $P$ value $\leq 0.05 S$ ). 
history of other diseases to be recruited under same study in order to evaluate the role of such GSTs enzymes in the association of HCV acute and chronic infection.

To evaluate and confirm the role of GSTs Null genotypes with the increased risk of $\mathrm{HCV}$ infection, the molecular assessment of GSTs genotyping was performed for GSTT1 and GSTM1. It was observed that among 56 positive patient's samples, GSTT1(-) were $21.05 \%$, GSTM1(-) $28.95 \%$ and those patient having GST's null genotypes were $23.69 \%$ however $26.3 \%$ of individuals among them showed normal GST pattern. This indicate that the GSTs isoform that most frequently deleted are GSTM1 as compare to GSTs null and GSTT1 isoform irrespective of the patient gender and age, acute and chronic infections. The close observations have been observed that GSTs may mutated during acute and chronic infection of HCV by some unknown mechanism which may lead to liver cirhosis and hepatocellular carcinoma, however incase of normal individuals no such pattern was observed [17]. Similarly GSTs genotyping also performed for the 20 PCR negative individuals but no such null genotyping was observed for those individuals.

Among male the observed GSTT1, GSTM1 and Null genotypes were $11.84 \%, 17.11 \%$ and $18.42 \%$ while $15.79 \%$ of the male were those having no such null genotypes were observed. Similarly in female the observed GSTT1, GSTM1 and Null genotypes were $9.21 \%, 11.84 \%$, and $5.26 \%$ while $10.53 \%$ of the

female were those in which no such null genotypes were observed (Figure. 2). Although males have higher incidence of $\mathrm{HCV}$ infections but pattern of GSTT1, GSTM1 and null genotypes showed similarity in both male and female, both the male and female had highest frequency of GSTM1 null genotype, followed by null GST genotype and GSTT1 in male. However, in female GSTT1 deletion was more common than null genotype. Gender difference seems to be irrelevant in these genes deletions or mutations in human. No such evidence was shown by the analysis that gene deletion shown some specificity towards either male or female.

Age factor is one of the prominent factors for GST to be associated with HCV infection and pathogenecity. Among the selected individuals for this study it was observed that individuals equal or more than $31^{\text {th }}$ years old were $81.58 \%$ had GSTT1, GSTM1 and GSTs Null genotypes by percentage of $18.42 \%$, $22.37 \%$ and $19.74 \%$ respectively whereas $21.05 \%$ of the individuals are those having normal GST genotypes, placed as normal with respect to GSTs. Similarly individuals which are younger than $31^{\text {th }}$ years were $18.42 \%$, had GSTT1, GSTM1 and GSTs Null genotypes by percentage of $2.63 \%, 6.58 \%$ and $3.95 \%$ while $5.26 \%$ individuals with normal GSTs pattern (Figure. 3). Our observation concluded that age factor have no visible impact on deciding fate of null genotypes nature, because in both patient, elder than and younger than 31 years have the highest frequency of GSTM1 (-), null genotypes followed by GSTT1 (-). Individuals both having age either below 31 years or above 31 years have null genotype in least frequency. However there are no specific GSTs isoform deletion has observed in both age groups. There is no prominent correlation yet reported regarding age and GSTs.

GSTs usually have observed to have relation with increased onset of disease, therefore in order to correlate both HCV acute and chronic infections with the GSTs, HCV positive patients were categorized into acute and chronic HCV patients. It was observed 
that there were total $39.29 \%$ acute infection patients GSTM1 null genotypes were $15.70 \%$ and GSTs Null genotypes were $6.54 \%$. However total HCV chronic infection were $60.71 \%$, among these GSTT1, GSTM1 and GSTs Null genotypes were 7.55\%, $13.25 \%$ and $17.15 \%$ respectfully (Figure 4 ). Like general GSTs mutation pattern, our observations indicated that due to acute infection the frequency of GSTM1 was the highest followed by GSTT1 deletion or mutation. The frequency of null genotype was low at acute stage, while individuals suffering from chronic infection were having the highest frequency of GSTs null genotypes followed by GSTM1 null genotype; however the patients with GSTT1 deletion or alteration were low in chronic infections. It seems that early infection causes only GSTM1 deletion or mutation while as infection proceeds the GSTT1 also get mutated by viral toxins. However in chronic infection both of the genes lost functionality. There are no such literature information are available about the relationship of the GSTs genotypes and HCV infection but some close observations have been reported [14].

\section{Conclusion and Recommendation}

From the ongoing research it has been concluded that $\mathrm{HCV}$ infection may cause the deletion or mutation of genes especially GSTM1 by some unknown mechanism and pathogenicity. At the earlier stage only single gene mutation occurs however with the progressesion of infection, mutation of both GSTT1 and GSTM1 isoforms occurs. The main achievement of this research was to conclude that increase in age increases chances of GSTM1 and GSTT1 malfunctioning. More frequent deletion occurs when infection enters into chronic stage. Similarly both male and female get GSTM1 mutations more frequently due to $\mathrm{HCV}$ infection. It has been among these GSTT1 null genotypes were 13.55\%, concluded that potential causes of these deletion and onset of $\mathrm{HCV}$ infection may be polluted water, tobacco addiction, low socioeconomically status (unhygienic environment) and increased viral load, because from our data most of the infected individuals were using tube well water and belong to low socioeconomic status and most of them were tobacco addicted. The main limitation of the study is the relatively small sample size. Further research will be needed to confirm whether these results generalize to general population through a large community based study. In addition, further studies are needed to correlate HCV infection with GSTT1 (-), GSTM1 (-) and null genotype to evaluate the true correlation of GST Null genotypes with age and infection status.

\section{Acknowledgment}

We are thankful to all patients and volunteers who took part directly and indirectly in this study especially to Dr. Muhammad Jamil (Assistant Professor) Department of Biotechnology \& Genetic Engineering, KUST for providing funds for this research. All authors declare no conflict in term of collaboration.

\section{References}

1. a b Jancovaa PP, Anzenbacherb $P$ and Anzenbacherovaa E (2010) Phase II drug metabolizing enzymes. Biomed. Pap Med Fac Univ Palacky Olomouc Czech Repub 154(2):103-116.

2. Sheehan D, Meade G, Foley VM and Dowd CA (2001) Structure, function and evolution of glutathione transferases: implications for classification of non-mammalian members of an 
ancient enzyme superfamily. Biochem J 360(1): $1-16$.

3. Hayes JD and Strange RC (2000) Glutathione Stransferase polymorphisms and their biological consequences. Pharmacology 61: 154-166.

4. Hayes JD, Flanagan JU and Jowsey IR (2005) Glutathione transferases. Annu Rev Pharmacol Toxicol 45: 51-88.

5. Ahmad W, Ijaz B, Javed FT, Kausar H, Sarwar MT, Gull S, Asad S, Shahid I, Hassan S (2011) HCV Genotype-Specific Correlation with Serum Markers: Higher Predictability for Genotype 4a. Virol J 8: 293-301.

6. Raja NS, Janjua KA (2008) Epidemiology of hepatitis $\mathrm{C}$ virus infection in Pakistan. J Microbiol Immunol Infect 41:4-8.

7. World Health Organization. Hepatitis (1997) Wkly Epidemiol Rec 72: 65-72.

8. Koike K and Miyosh H (2006) Oxidative stress and hepatitis $\mathrm{C}$ viral infection. Hepatol Res 34: 65-73.

9. Hartley DP, Ruth JA and Petersen DR (1995) The hepatocellular metabolism of 4hydroxynonenal by alcohol dehydrogenase, aldehyde dehydrogenase, and glutathione Stransferase. Arch Biochem Biophys 316: $197-$ 205.

10. Luckey SW and Petersen DR (2001) Metabolism of 4-hydroxynonenal by rat Kupffer cells. Arch Biochem Biophys 389: 77-83.
11. Chen J, Schenker S and Henderson GI (2002) 4Hydroxynonenal detoxification by mitochondrial glutathione S-transferase is compromised by shortterm ethanol consumption in rats. Alcohol Clin Exp Res 26: 1252-8.

12. Xu S, Wang YP, Roe B and Pearson WR (1998) Characterization of the human class mu glutathione S-transferase gene cluster and the GSTM1 deletion. J Biol Chem 273: 3517-27.

13. Boya P, Pena ADL and Beloqui O (1999) Antioxidant status and glutathione metabolism in peripheral blood mononuclear cells from patients withchronic hepatitis C. J Hepatol 31: 808-14.

14. a b Martinez CE, Martin JM, Ladero O, Herraez L, Ortega C, Taxonera A, Suarez MD, Rubio J and Agundez (2007) GSTT1 and GSTM1 Null Genotypes May Facilitate Hepatitis C Virus Infection Becoming Chronic. The Journal of Infectious Disease 195: 1320-3.

15. Arbag HT, Cora H, Acar K, Ozturk B, Ulusoy and F Sari (2006) Lack of association between the glutathione-s-transferase genes (GSTT1 andGSTM1) and nasal polyposis. Rhinology 44: 14-18.

16. Settheetham-Ishida W, Singto Y and Yuenyao P (2004) Contribution of epigenetic risk factors but not p53 codon 72 polymorphism to the development of cervical cancer in Northeastern Thailand. Cancer Letters 210: 205-11.

17. Song K, Yi J, Shen X and Yu CY (2012) Genetic Polymorphisms of Glutathio ne S-Transferase Genes GSTM1, GSTT1 and Risk of Hepatocellular Carcinoma. PLOS ONE 7(11). 